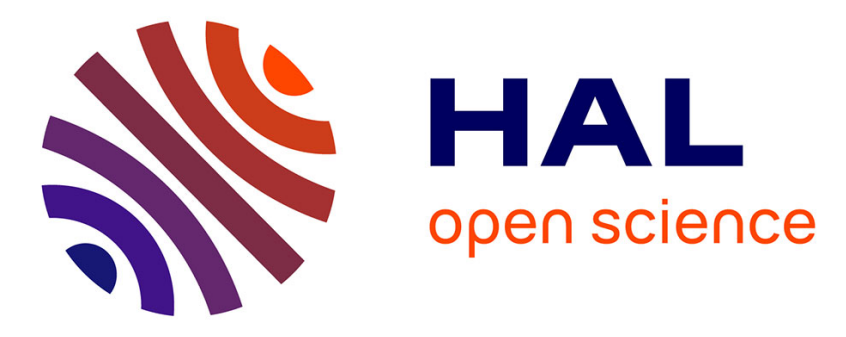

\title{
Simple Engineering of Polymer-Nanoparticle Hybrid Nanocapsules
}

Flavien Sciortino, Gérald Casterou, Pierre-Antoine Eliat, Marie-Bérengère Troadec, Cedric Gaillard, Soizic Chevance, Myrtil L. Kahn, Fabienne Gauffre

\section{- To cite this version:}

Flavien Sciortino, Gérald Casterou, Pierre-Antoine Eliat, Marie-Bérengère Troadec, Cedric Gaillard, et al.. Simple Engineering of Polymer-Nanoparticle Hybrid Nanocapsules. ChemNanoMat, 2016, 2 (8), pp.796-799. 10.1002/cnma.201600155 . hal-01395464

\section{HAL Id: hal-01395464 https://hal-univ-rennes1.archives-ouvertes.fr/hal-01395464}

Submitted on 4 May 2020

HAL is a multi-disciplinary open access archive for the deposit and dissemination of scientific research documents, whether they are published or not. The documents may come from teaching and research institutions in France or abroad, or from public or private research centers.
L'archive ouverte pluridisciplinaire HAL, est destinée au dépôt et à la diffusion de documents scientifiques de niveau recherche, publiés ou non, émanant des établissements d'enseignement et de recherche français ou étrangers, des laboratoires publics ou privés. 


\title{
Simple Engineering of Polymer-Nanoparticle Hybrid Nanocapsules
}

\author{
Flavien Sciortino ${ }^{[a]}$, Gérald Casterou ${ }^{[a, b]}$, Pierre-Antoine Eliat ${ }^{[c]}$, Marie-Bérengère Troadec ${ }^{[d]}$, Cédric \\ Gaillard $^{[e]}$, Soizic Chevance ${ }^{[a]}$, Myrtil L Kahn ${ }^{*[b]}$, Fabienne Gauffre*[a]
}

\begin{abstract}
A general method to generate hybrid hollow capsules is reported. The process is based on the stabilization of solvent droplets by nanoparticles, in macroscopically miscible mixtures of THF and water. After addition of a crosslinking polymer and removal of the solvent core, capsules of diameter ca $100 \mathrm{~nm}$ are obtained. This novel strategy does not require the use of block copolymers. In contrast, most methods reporting the formation of hybrid nanocapsules incorporate nanoparticles into block-copolymer polymersomes or use nanoparticles tethered with block-copolymers. The nanocapsules were characterized using a full set of techniques including nanoparticle tracking analysis, electron microscopy and liquid phase atomic force microscopy. Our results show that the vesicular shape of the nanocapsules is templated by the liquid droplets. Nanocapsules were prepared from Quantum Dots, gold nanoparticles, superparamagnetic iron oxide nanoparticles and mixtures of particles. The entrapment of a fluorescent dye was also demonstrated. Thus, nanocapsules with dual properties (e.g. magnetic and fluorescent) are easily obtained. Interestingly, the magnetic nanocapsules enable Magnetic Resonance Imaging contrast enhancement of tumors in vivo.
\end{abstract}

The interest in forming assemblies of inorganic nanoparticles and polymers is manifold. On the one hand, the nanoparticles bring optical or magnetic properties which can be used for sensing, imaging, or heating, whereas on the other hand the polymers provide functions such as response to external stimuli $(\mathrm{pH}$, temperature), stealthiness and molecular recognition. ${ }^{[1]}$ Although such assemblies held much promise as multifunctional platforms for nanomedicine, the control of their size and morphology remains a daunting challenge. A variety of strategies have been proposed to elaborate nanoparticle-polymer assemblies. ${ }^{[2]}$ For instance, the in situ synthesis of nanoparticles within linear or

[a] F. Sciortino, Dr. G. Casterou, Dr S. Chevance, Dr. F. Gauffre Institut des Sciences Chimiques de Rennes, UMR 6226 CNRS, Université de Rennes 1

263 av. Général Leclerc, 35042 Rennes Cedex, France E-mail: fabienne.gauffre@univ-rennes1.fr

[b] Dr. G. Casterou, Dr. M. L. Kahn Laboratoire de Chimie de Coordination UPR8241 CNRS, 205 rte de Narbonne, Toulouse cedex 04, France.

[c] Dr. P-A. Eliat PRISM-Biosit,

Université de Rennes 1, CNRS-UMS 3480, INSERM UMS 018, Rennes, France

[d] Dr. M-B. Troadec IGDR, UMR 6290 CNRS, Université de Rennes 1, 2 av. du Prof. Bernard, CS 34317, 35043 Rennes Cedex, France

[e] Dr. C. Gaillard INRA, La Géraudière 44316 Nantes cedex 3, France hyperbranched polymers is generally a convenient one-pot procedure in which the polymer promotes the nucleation of the particle while preventing uncontrolled clustering. ${ }^{[3,4]}$ Alternatively, nanoparticles can be encapsulated within block-copolymer micelles ${ }^{[5,6]}$ or coassembled with polymers via nanoprecipitation or electrostatic interactions. ${ }^{[7,8]}$ Recently, many efforts have been devoted to the elaboration of hybrid nanoparticle-polymer nanocapsules, which offer the possibility to encapsulate and potentially release drugs. Thus, polymersomes incorporating inorganic nanoparticles into their membranes were fabricated via the cooperative selfassembly of amphiphilic block-copolymers and nanoparticles. ${ }^{[9]}$ Alternatively, preformed polymersomes were armored with a layer of nanoparticles via electrostatic interactions. ${ }^{[9]}$ It was also demonstrated that inorganic nanoparticles tethered with blockcopolymers self-assemble into superstructures such as clusters and vesicles. ${ }^{[10-14]}$ All these strategies have in common the use of block copolymers which direct the self-assembly.

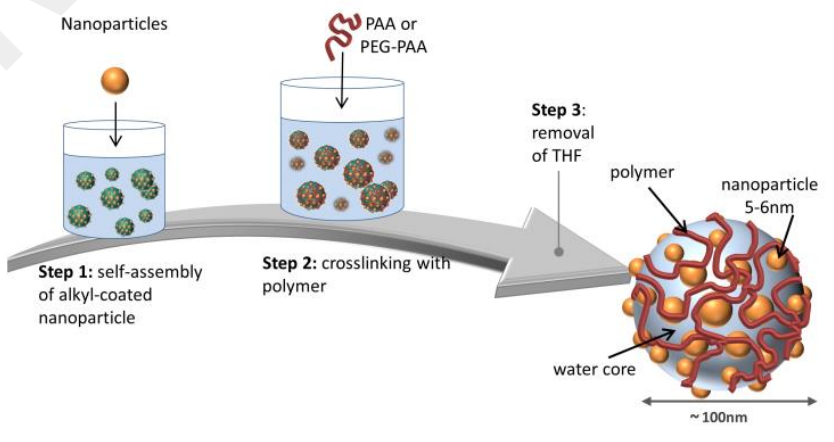

Figure 1. Process of elaboration of the hybrid nanocapsules.

We report here for the first time a general process to generate hybrid hollow capsules without the need for block-copolymers. The formation of the vesicles is templated by solvent droplets in mixtures of water and THF. Indeed, although water and THF are macroscopically miscible, nanodroplets are formed in the water rich region, which we used as templates for the self-assembly of the nanoparticles. After crosslinking the nanoparticles with a suitable polymer the solvent core can be removed. The resulting vesicles possess a "composite" or "hybrid" nanoparticle-polymer shell. In the following we will refer to them as hybridosomes. Based on these findings, we elaborated hybridosomes from a variety of nanoparticles and evaluated the in vivo MRI efficiency of hybridosomes made from superparamagnetic iron oxide nanoparticles (SPION). 


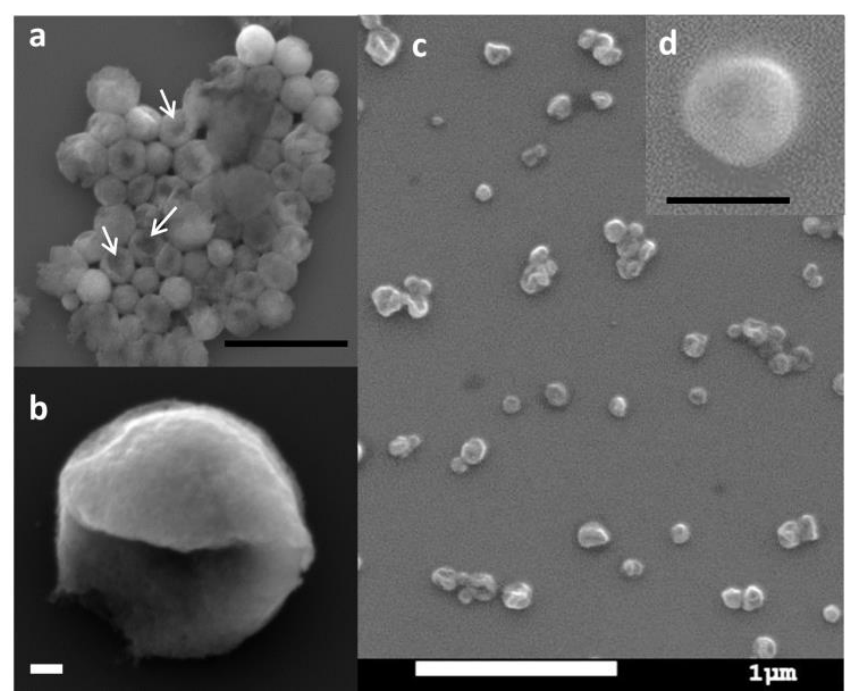

Figure 2. SEM images of (a-b) assemblies of SPIONs in water/THF mixtures; and (c-d) magnetic hybridosomes. (a) $[\mathrm{Fe}]=65 \mu \mathrm{g} / \mathrm{ml}$; (b) $[\mathrm{Fe}]=80 \mu \mathrm{g} / \mathrm{ml}$ (c-d) $[\mathrm{Fe}]=25 \mu \mathrm{g} / \mathrm{ml}$; polymer: PAA450k. Bars: $(\mathrm{a} ; \mathrm{c})=1 \mu \mathrm{m} ;(\mathrm{b} ; \mathrm{d})=100 \mathrm{~nm}$. White arrows indicate deflated capsules.

We have all learned from our textbooks that water is miscible in all proportions with THF. However, dynamic light scattering (DLS) and small angle neutron scattering measurements clearly evidence fluctuations of concentrations for specific compositions of water/THF mixtures. Such fluctuations have also been reported for mixtures of water with other "miscible" solvents such as acetone or alcohols, ${ }^{[15-17]}$ the nature of which is still in debate. ${ }^{[18]}$ It has been suggested that they could be either solute droplets ${ }^{[15]}$ or air bubbles. ${ }^{[19]}$ Using Nanoparticle Tracking Analysis (NTA) and analytical centrifugation, Sedlak et al. have provided convincing evidence of the existence of droplets of about $100 \mathrm{~nm}$ in diameter in various mixtures of miscible liquids. ${ }^{[15]}$

Inspired by these results, we hypothesized that such droplets could template the formation of nanoparticle shells, similarly to Pickering emulsions. Nanoparticles are not amphiphilic but strongly adsorb at interfaces between polar and non-polar liquid. ${ }^{[20]}$ We first looked for solvent droplets in water/THF mixtures in a wide range of compositions ( $\varphi_{w}=0.05-0.9$, water vol. fraction). Time fluctuations were clearly measured using DLS, with faster dynamics as more water was added (Figure S1). Particularly, a mode corresponding to the Brownian motion of small objects (length scale $100 \mathrm{~nm}$ ) appeared for compositions close to $\varphi_{w}=0.8$. Note that DLS is sensitive to any fluctuation and that the dynamic modes that were measured do not necessarily correspond to droplets. To verify the presence of droplets, NTA was undertaken for compositions with the fastest correlation modes $\left(\varphi_{w}=0.8-0.9\right)$. The results clearly evidenced finite structures with a hydrodynamic diameter $D_{h}=110 \pm 35 \mathrm{~nm}$ (Figure S2).

Figure 3. AFM of SPIONs hybridosomes in aqueous solution. (a-a') 3-D topography; (b-b') lateral views; (c) TEM image of the corresponding sample. $[\mathrm{Fe}]=65 \mu \mathrm{g} / \mathrm{ml}$; PAA450k.

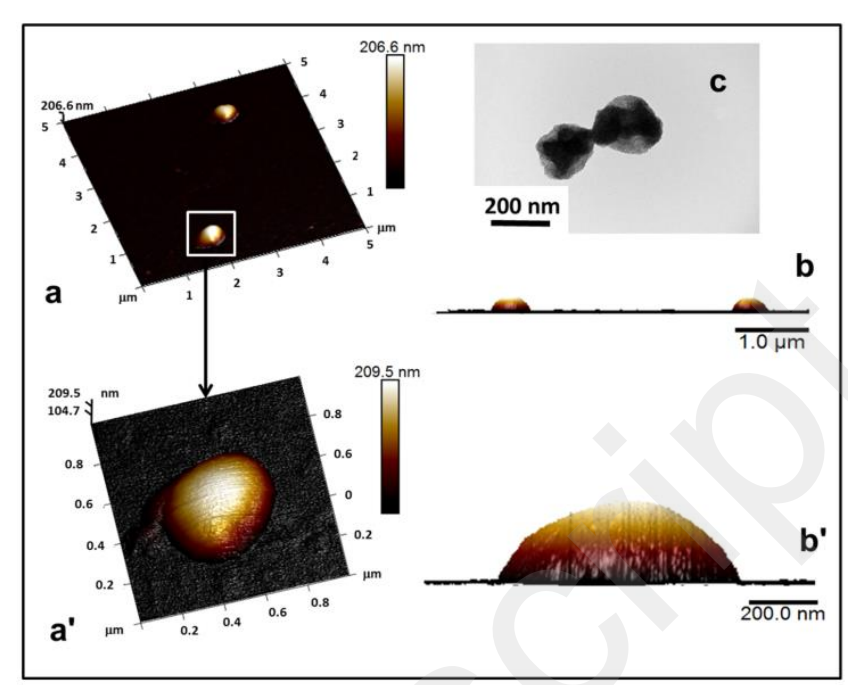

We then investigated the water/THF/NP (NP = nanoparticles) mixtures using dodecanthiol-coated gold nanoparticles (AuNP) (Figure S3a). In the presence of small or moderate amounts of water $\left(\varphi_{w}=0.05\right.$ to 0.4$)$, a bad solvent for the NPs, they rapidly aggregate and sediment as expected. However, stable suspensions were found at higher water contents $\left(0.5<\varphi_{w}<0.9\right)$, the most stable samples $\left(\varphi_{w}=0.8\right)$ showing no sign of sedimentation over more than one year. A similar behavior was found for $\gamma-\mathrm{Fe}_{2} \mathrm{O}_{3}$ (maghemite) SPION (Figure S3b). TEM revealed relatively ill-defined structures for $\varphi_{w}=0.42$, whereas regular spherical aggregates with a diameter of ca $100 \mathrm{~nm}$ were obtained at $\varphi_{w}=0.8$. DLS measurements clearly evidenced the stabilization of the smaller droplets at the expenses of larger ones in the presence of the nanoparticles (Figure S4).

Based on these findings, we proposed a general strategy to elaborate stable nanoparticle-polymer capsules (Figure 1). Starting from THF solutions of hydrophobic nanoparticles the selfassembly is triggered by adding water to a final volume fraction $\varphi_{w}=0.8$. This causes the nanoparticles to gather at the surface of solvent droplets (step 1). On addition of a suitable polymer, a crosslinked network of nanoparticles and polymers forms around the droplets (step 2). Finally, the removal of the solvent yields the hybridosomes (step 3).

As a proof of principle, we firstly elaborated magnetic hybridosomes from SPIONs. Assemblies of SPIONs are of particular interest in a wide range of applications including MRI ${ }^{[21]}$ and protein separation, ${ }^{[22]}$ among others. However, due to their small size, single SPIONs are difficult to collect under magnetic field and make poor MRI contrast agents. A large number of theoretical and experimental studies have demonstrated that clustering of SPIONs increase the transversal relaxivity $r_{2}$, thereby enhancing the efficiency as $T_{2}$ contrast agent. ${ }^{[23-26]}$

Figure 4. TEM of (a) SPION hybridosomes ([Fe] $=50 \mu \mathrm{g} / \mathrm{mL}$; polymer: PEGPAA); (b) mixed hybridosomes from AuNP and SPIONs, the AuNP appear with darker contrast ([Fe]=[Au] $=26 \mu \mathrm{g} / \mathrm{mL}$; polymer: PAA450k), (c) SEM of (CdSe@ZnS)QD-hybridosomes. ([QD]=26 $\mathrm{g} / \mathrm{mL}$, PAA450k) Bars: (a) 500 nm; (b) $50 \mathrm{~nm}$; (c) $100 \mathrm{~nm}$. 


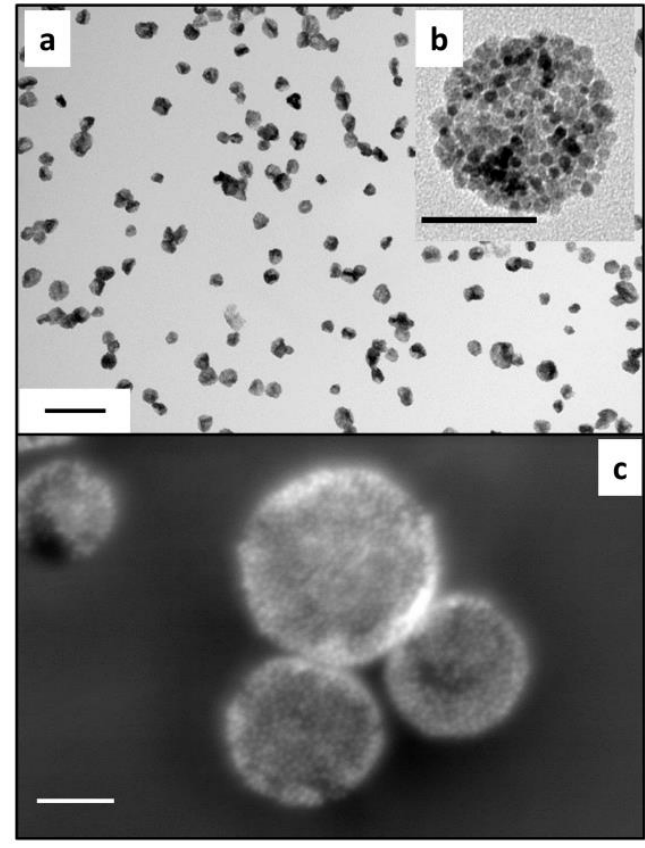

Magnetic hybridosomes were prepared following the scheme depicted in Figure 1. Poly(acrylic acid) (PAA) was selected as the polymer for its ability to link to iron oxide, via the multiple carboxylic acid functions. ${ }^{[27]}$ Alternatively, we used a poly(ethylenglycol)-co-PAA diblock copolymer to prepare PEGylated hybridosomes (Figure 4a). ${ }^{[28]}$ The SEM analysis of the dried samples at step 1 and step 2 clearly evidenced deflated capsules (indicated by arrows, Figure 2) together with spherical ones. In their hydrated state, the hybridosomes adopt a swollen shape as shown by AFM analysis in water (Figure 3). To confirm their hollow structure, we imaged the hybridosomes using TEM tomography (Figure 5, Movie S1). Due to the small size of the pristine nanoparticles, it is difficult to identify them individually on the 3-dimensional reconstruction. However, the hollow structure is clearly confirmed both by the animated tilted projection (Movie S1) and the 3D-reconstruction. The templating effect of droplets clearly appears when a lower amount of nanoparticles is used as in Figure S5. In this case, the partial coverage of the surface by nanoparticles underlines the shape of the initial droplet. All together, these analyses strongly support the hypothesis that the hybridosomes are soft hollow capsules. The hybridosomes were easily separated from the supernatant by applying a magnet and then resuspended without any noticeable aggregation.

Mixed hybridosomes of AuNP and SPIONs as well as hybridosomes from QD were also prepared (Figure 4b-c). TEM (Figure 4b) and EDX (Figure S6) analysis of the mixed AuNP/SPION hybridosomes both confirm the co-localization of gold and iron. Since the PAA does not link to the AuNP, a collapse of the AuNP within the core of the hybridosomes on removal of THF cannot be excluded. However, the blue resonance characteristic of coupled AuNp was not observed.
Figure

of the 3density

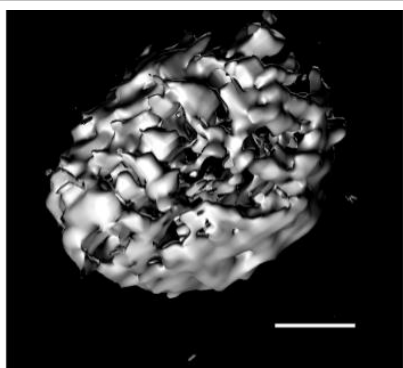

5. Top view dimensional map

reconstructed from TEM-tomography of magnetic hybridosomes (SPION $[\mathrm{Fe}]=50 \mu \mathrm{g} / \mathrm{mL}$; polymer: PAA450k). Scale bar $=20 \mathrm{~nm}$

SPIONs hybridosomes could also be loaded with a fluorescent BODIPY dye, simply by dissolving the dye in the THF solution at the initial stage of the process, yielding dual magnetic and fluorescent features (Figure S7).

The transversal relaxivities $r_{2}$ of the magnetic hybridosomes were found comparable to those of commercial contrast agents such as Feraspin ${ }^{\mathrm{TM}}$ in vitro (Figure S8). Therefore, we evaluated their imaging ability for in vivo Magnetic Resonance (MR) using a mouse model. The $\mathrm{T}_{2}{ }^{*}$-weighted images show a significant extinction of the liver signal after retro-orbital intravenous injection (Figure S9). Interestingly, AuNP/SPION hybridosomes also yielded significant contrast, which augurs interesting prospects for theranostic with MR-imaging and gold-based thermotherapy (Figure 6a-b). A further beneficial effect is the increased tumor detection (Figures 6c-d). We evaluated the in vitro cytotoxicity (Figure S10) to HEK cells for hybridosomes made from two types of particles (SPION and SPION/AuNp) and stabilized with the two polymers used in this study (PAA450k and PEG-PAA). After $48 \mathrm{~h}$ of exposure at an iron concentration of $100 \mu \mathrm{g}$ per 106 cells, both SPION/PAA450k and SPION/PEG-PAA hybridosomes show no detectable toxicity. Mixed SPION/AuNP hybridosomes stabilized with PAA450k show only a slight toxicity, with $85 \%$ of viability relative to the control, despite the high metal concentration ([Fe] $=[\mathrm{Au}]=100 \mu \mathrm{g}$ per 106 cells).
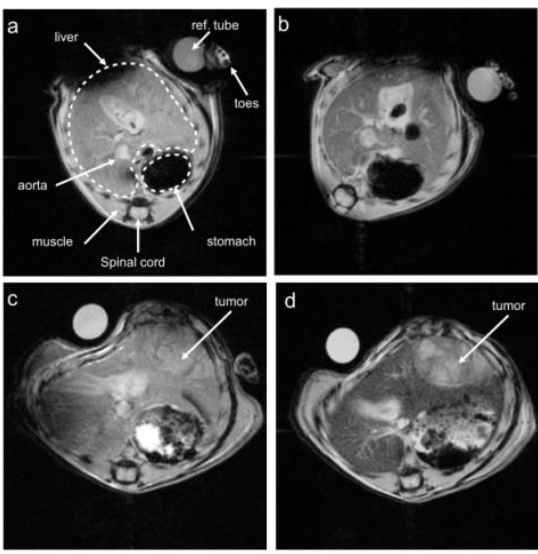

Figure 6 .

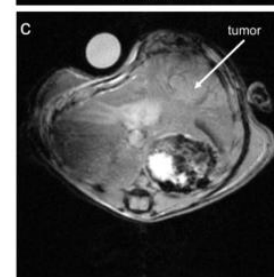

weighted

images of healthy $(a-b)$ and tumor bearing $(c-d)$ mice acquired before $(a ; c)$ and at $30 \mathrm{~min}(\mathrm{~b} ; \mathrm{d})$ after injection of the AuNP/SPION hybridosomes (0.5 to 0.9 $\mathrm{mg} / \mathrm{kg}$ ).

In summary, based on the observation that THF and water mixtures contain droplets of well-defined size, we propose a new strategy to form hybrid nanoparticle-polymer nanocapsules, using these droplets as templates. In contrast with previous methods, 
this novel strategy does not require the use of amphiphilic blockcopolymers and produces vesicles of smaller size. We demonstrated that such vesicles can be readily obtained from nanoparticles of various chemical composition provided that the polymer crosslinks the nanoparticles as it is the case for PAA with iron oxide or with CdSe@ZnS. Particularly, SPION containing hybridosomes provide efficient MRI contrast enhancement, enabling the imaging of tumor areas at low dose and show low if any toxicity. Not only does this study demonstrate a versatile method for the facile engineering of well-defined assemblies with combined plasmonic, magnetic and/or fluorescent properties, but it also brings into light the role of preformed solvent droplets in the formation of vesicular aggregates.

\section{Acknowledgements}

The authors thank O. Mongin (ISCR), M. Destarac (Univ. Toulouse) and O. Musso (INSERM) for providing BODIPY, PEGPAA and the tumor bearing mice. We are grateful to BIOSITUniversity of Rennes1 for financial support and to MRic (A. Burel), CMEBA (V. Dorcet) and THEMIS for analysis.

Keywords: nanoparticles $•$ polymer $\bullet$ self-assembly $\bullet$ Magnetic Resonance Imaging • nanocapsules

[1] K. T. Kim, S. Meeuwissen, R. J. Nolte, J. van Hest, Nanoscale 2010, 2, 844-58.

[2] M. Hood, M. Mari, R. Muñoz-Espí, Materials 2014, 7, 4057-4087.

[3] S. Saliba, C. Valverde-Serrano, J. Keilitz, M. Kahn, C. Mingotaud, R. Haag, J.-D. Marty, Chem. Mater. 2010, 22, 6301.

[4] J. Keilitz, M. R. Radowski, J. Marty, R. Haag, F. Gauffre, Chem. Mater. 2008, 20, 2005-2007.

[5] S. Förster, T. Plantenberg, Angew. Chem. Int. Ed. Engl. 2002, 41, 689714

[6] E. Pöselt, S. Fischer, S. Foerster, H. Weller, Langmuir 2009, 25, 13906-13913.

[7] N. Pinkerton, M. Gindy, V. Calero-Diaz del Castillo, T. Wolfson, R. Pagels, D. Adler, D. Gao, S. Li, R. Wang, M. Zevon, et al., Adv. Healthc. Mater. 2015, 4, 1376-1385.
[8] J.-F. Berret, K. Yokota, M. Morvan, R. Schweins, J. Phys. Chem. B 2006, 110, 19140-6.

[9] R. Chen, D. Pearce, S. Fortuna, D. Cheung, S. Bon, J. Am. Chem. Soc. 2011, 133, 2151-3.

[10] Y. Guo, S. Harirchian-Saei, C. M. S. Izumi, M. G. Moffitt, ACS Nano 2011, 5, 3309-18

[11] E. Pöselt, H. Kloust, U. Tromsdorf, M. Janschel, C. Hahn, C. Maßlo, H. Weller, ACS Nano 2012, 6, 1619-1624.

[12] J. He, X. Huang, Y. C. Li, Y. Liu, T. Babu, M. A. Aronova, S. Wang, Z Lu, X. Chen, Z. Nie, J. Am. Chem. Soc. 2013, 135, 7974-7984.

[13] J. Lin, S. Wang, P. Huang, Z. Wang, S. Chen, G. Niu, W. Li, J. He, D. Cui, G. Lu, et al., ACS Nano 2013, 7, 5320-5329.

[14] Y. Liu, J. He, K. Yang, C. Yi, Y. Liu, L. Nie, N. M. Khashab, X. Chen, Z. Nie, Angew. Chemie - Int. Ed. 2015, 15809-15812.

[15] M. Sedlák, D. Rak, J. Phys. Chem. B 2013, 117, 2495-504.

[16] C. Yang, W. Li, C. Wu, J. Phys. Chem. B 2004, 108, 11866-11870.

[17] Z. Li, H. Cheng, J. Li, J. Hao, L. Zhang, B. Hammouda, C. C. Han, J. Phys. Chem. B 2011, 115, 7887-95.

[18] A. Häbich, W. Ducker, D. E. Dunstan, X. Zhang, J. Phys. Chem. B 2010, 114, 6962-6967.

[19] F. Jin, J. Ye, L. Hong, H. Lam, C. Wu, J. Phys. Chem. B 2007, 111, 2255-61.

[20] B. P. Binks, J. Colloid Interface Sci. 2002, 7, 21-41.

[21] H. Shokrollahi, A. Khorramdin, G. Isapour, J. Magn. Magn. Mater. 2014 369, 176-183.

[22] L. Xie, S. Ma, Q. Yang, F. Lan, Y. Wu, Z. Gu, RSC Adv. 2014, 4, 10551061.

[23] P. M. Peiris, E. Schmidt, M. Calabrese, E. Karathanasis, PLoS One 2011, 6, e15927.

[24] Y. Lin, S. Wang, Y. Zhang, J. Gao, L. Hong, X. Wang, W. Wu, X. Jiang, J. Mater. Chem. B 2015, 3, 5702-5710

[25] J.-F. Berret, N. Schonbeck, F. Gazeau, D. El Kharrat, O. Sandre, A Vacher, M. Airiau, J. Am. Chem. Soc. 2006, 128, 1755-1761.

[26] Q. L. Vuong, J. Berret, J. Fresnais, Y. Gossuin, O. Sandre, Adv. Healthc. Mater. 2012, 1, 502-512.

[27] J. Fresnais, M. Yan, J. Courtois, T. Bostelmann, A. Bée, J.-F. Berret, J. Colloid Interface Sci. 2013, 395, 24-30.

[28] H. Bagaria, K. Yoon, B. Neilson, V. Cheng, J. H. Lee, A. Worthen, Z. Xue, C. Huh, S. Bryant, C. Bielawski, et al., Langmuir 2013, 29, 3195206. 
Hybridosomes. Although water and THF are known as miscible solvent, their mixtures contain droplets that template the self-assembly of nanoparticles and polymers. This strategy was successfully applied to elaborate hollow nanocapsules $(\sim 100 \mathrm{~nm})$ with a hybrid shell made of crosslinked polymers and nanoparticles. Magnetic nanocapsules proved to be efficient as in vivo MRI contrast agent for tumor imaging.
Flavien Sciortino, Gérald Casterou, Pierre-Antoine Eliat, Marie-Bérengère Troadec, Cédric Gaillard, Soizic Chevance, Myrtil L Kahn, * Fabienne Gauffre*

Simple Engineering of PolymerNanoparticle Hybrid Nanocapsules 
Additional Author information for the electronic version of the article.

F. Gauffre: $\quad$ 0000-0003-2835-3556

Author: ORCID identifier

Author: $\quad$ ORCID identifier

\section{Content}

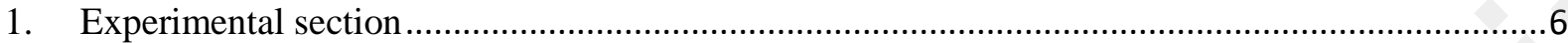

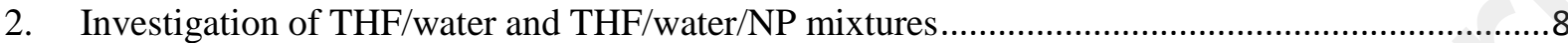

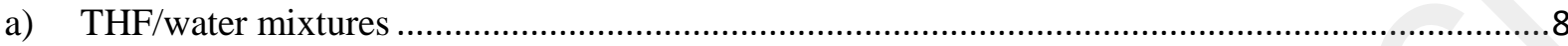

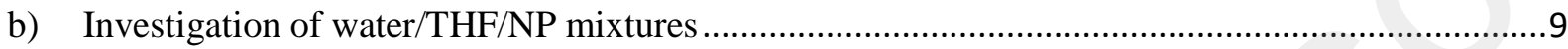

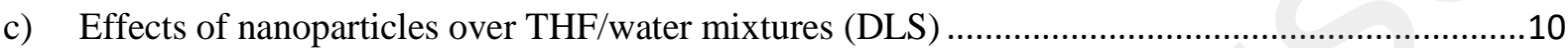

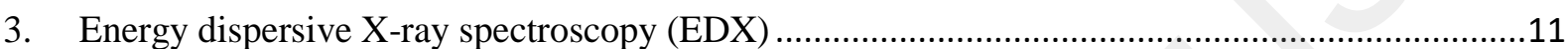

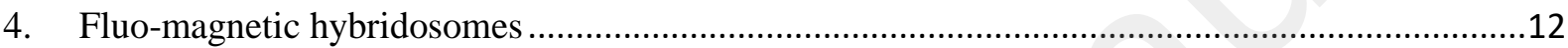

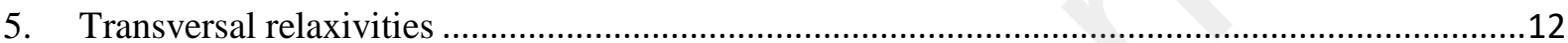

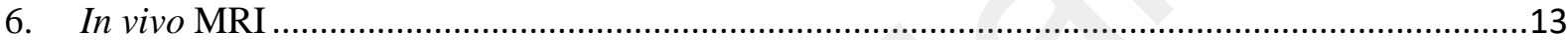

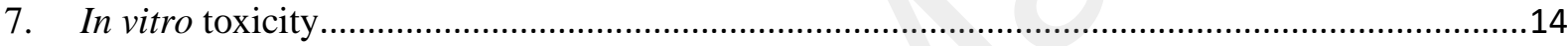

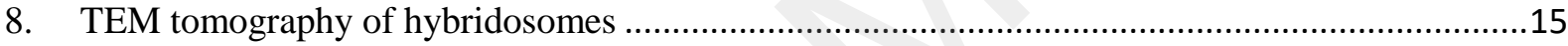

\section{Experimental section}

Materials. Octylamine was purchased from Sigma-Aldrich and stored as received in an argon-filled glove box. $\left\{\mathrm{Fe}\left[\mathrm{N}\left(\mathrm{SiMe}_{3}\right)_{2}\right]_{2}\right\}$ (purchased from Nanomeps) was stored as received in the fridge of the glove box. $5.4 \mathrm{~nm}$ dodecanthiol coated gold NP (AuNP) were purchased from Nanocomposix. Tetrahydrofuran (VWR) was used as received. Poly(acid acrylic) PAA (1.88k or 450k) was purchased from Aldrich. The BODIPY 1 was kindly provided by O. Mongin (ISCR) and synthesized following a reported procedure ${ }^{[1]}$ Octadecylamine-coated CdSe@ZnS core-shell quantum dots in powder form were purchased from Sigma-Aldrich. The MRI constrast agent Feraspin XXL ${ }^{\mathrm{TM}}$ was purchased from Miltenyi Biotec.

SPION synthesis. The synthesis of the maghemite $\left(\gamma-\mathrm{Fe}_{2} \mathrm{O}_{3}\right)$ SPIONs was achieved via hydrolysis of an organometallic $\mathrm{Fe}(\mathrm{II})$ precursor following a recently reported procedure. ${ }^{[1]}$ The reaction was achieved in pure octylamine and in mild conditions of pressure and temperature. $\mathrm{H}_{2} \mathrm{O}$ was deoxygenated by bubbling argon 30 mins before the reaction and stored under argon. The precursor $\left\{\mathrm{Fe}\left[\mathrm{N}\left(\mathrm{SiMe}_{3}\right)_{2}\right]_{2}\right\}$ and octylamine ( 2 eq. to the precursor) were introduced in vials in a glovebox under argon. After a dark homogeneous liquid was obtained the vials were placed in a closed reactor, removed from the glovebox, and set to $40^{\circ} \mathrm{C}$. After $15 \mathrm{~min}, \mathrm{H} 2 \mathrm{O}$ (2.1 eq.) was introduced in the reactor. The reaction was stopped after 3 days by putting the reactor under vacuum. The mixture was then exposed to air and dispersed in THF. The solution was then centrifuged at $19000 \mathrm{rcf}$ for $10 \mathrm{~min}$ and the pellet discarded. The mean diameter measured from TEM analysis was $5.56 \mathrm{~nm}$ (0.60 nm standard deviation). After air oxidation, 
superparamagnetic magnetite nanocrystals with a mean diameter $\mathrm{d}=5.5 \mathrm{~nm}$ and a narrow size distribution (standard deviation $1.0 \mathrm{~nm}$ ) are obtained. ${ }^{[1]}$

Elaboration of the hybridosomes. The hybridosomes were prepared by adding water to a THF solution containing the hydrophobic NP, in order to reach the composition $\varphi w=0.8$, promptly followed by vortexing (total metal concentration $\mathrm{C}=26-66 \mu \mathrm{g} / \mathrm{mL})$. After ca $15 \mathrm{~h}$, PAA $(2.1 \mathrm{mMol} . \mathrm{L}-1$ in AA unit) or PEG-PAA (2.7 mMol.L-1, in AA unit) were added to the mixture. The THF was then totally removed by slow evaporation $\left(15 \mathrm{~h}\right.$ at $\left.40^{\circ} \mathrm{C}\right)$, after which the remaining volume was ca $500 \mu \mathrm{L}$. It was verified that after this treatment, no traces of THF could be detected by $1 \mathrm{H}$ NMR.

Nanoparticle Tracking Analysis (NTA) and Dynamic Light scattering (DLS). NTA was carried out with a Nanosight LM10 device system equipped with a $40 \mathrm{~mW}$ laser working at $\lambda=638 \mathrm{~nm}$. Video sequences were recorded via a CCD camera operating at 30 frames per second and evaluated via the NANOSIGHT NTA 2.0 Analytical Software Suite. A blank NTA measurement with pure water was performed to exclude a possible contamination with scatterers. Calculations of the hydrodynamic diameters were achieved taking $1.22 \mathrm{cP}$ as the viscosity of THF/water mixture at $\varphi_{\mathrm{w}}=0.8$, from Nayak et al. ${ }^{[2]}$ DLS measurements were performed by using a NanoZS Instrument (Malvern) with Contin analysis to yield the size distribution by intensity.

Scanning Electronic Microscopy (SEM). A few microliters of a solution of hybridosomes were dropped onto a polished silicium wafer. Observation of sample was done with a JEOL 7100f ttls SEM at $10 \mathrm{kV}$ equipped with a tungsten tip and a Everhart-Thornley detector.

Transmission Electronic Microscopy (TEM). A 200mesh copper grid, covered with a carbon/formvar film was dipped into the solution of hybridosomes (at $[\mathrm{Fe}]=1 \mathrm{mM}$ ) and allowed to dry in ambient conditions. The samples were examined using a JEOL 1400 TEM operating at $120 \mathrm{kV}$ and equipped with a GATAN Orius 1000 camera or alternatively a JEOL 2100 LaB6 equipped with a GATAN Orius 200D and a SDD detector for Energy-Dispersive X-ray Spectroscopy (EDS) analysis. For TEM tomography a GATAN dual orientation 927 tomography sample holder was used. Tilted projection images were recorded at $1^{\circ}$ tilt intervals from $-50^{\circ}$ to $+30^{\circ}$ and we used Digital micrograph software to convert the TEM projections into the 3 -dimensional reconstructed image.

Atomic Force Microscopy (AFM). Samples were imaged in fluid over an area of $500 \mathrm{~nm}^{2}$ to $20 \mathrm{~mm}^{2}$ at 0.2 to $0.5 \mathrm{~Hz}$ operated in PeakForce Tapping mode on a BioScope Catalyst AFM (Bruker AXS, Santa Barbara, CA) using a small volume liquid cell and a set-point of around 1-2 nN. SCANASIST-Fluid AFM tips (Bruker AFM Probes, Camarillo, CA) with a nominal spring constant, resonant frequency and radius of $0.7 \mathrm{~N} / \mathrm{m}, 150 \mathrm{kHz}$ and $20 \mathrm{~nm}$ respectively, were used for each experiment. AFM data were analyzed using the Nanoscope Analysis 1.5 software (Bruker AXS, Santa Barbara, CA).

Relaxivity measurements. Relaxivity measurements of hybridosomes were performed by measuring $\mathrm{T}_{2}$ values at various iron concentrations in water (determined by ICP-OES). Acquisitions were performed at $4.7 \mathrm{~T}$ on a Bruker Biospec 47/40 horizontal MRI system equipped with a $72 \mathrm{~mm}$ inner diameter volume transmit/receive quadrature coil. The $\mathrm{T}_{2}$ values were measured by using a multi-echo spin echo sequence with 16 echoes, an echo time spacing of $8 \mathrm{~ms}$, and a repetition time (TR) of 2000 $\mathrm{ms}$. The $\mathrm{T}_{2}$ maps were generated with ImageJ using the MRI analysis Calculator V1 by fitting the signal decay to a monoexponential function. Other imaging parameters were an image matrix size of $128 \mathrm{x}$ 128, a field of view (FOV) of $51.2 \mathrm{~mm}$, a slice thickness of $3 \mathrm{~mm}$, and one signal average per phaseencoding step. The measurements were performed at room temperature $\left(20.0 \pm 0.5^{\circ} \mathrm{C}\right)$. For each sample, a circular region of interest (ROI) was drawn from which $\mathrm{T}_{2}$ maps and then the mean $\mathrm{T}_{2}$ and standard deviations were calculated.

Cytotoxicity. HEK cells were grown in Dulbecco's modified Eagle medium (DMEM) supplemented with $10 \%$ fetal calf serum and $1 \%$ antibiotics. The hybridosomes were dialyzed against cell culture medium for $4 \mathrm{~h}$ prior to cell exposure. The cells were then exposed to hybridosomes $(100 \mu \mathrm{g}$ of iron per 
106 cells) for $48 \mathrm{~h}$ at $37^{\circ} \mathrm{C}$ in cell culture medium. Viability assays were carried out by measuring MTS (3-(4,5-dimethyl-2-yl)-5-(3-carboxymethoxyphenyl)-2-(4-sulfophenyl)-2H-tetrazolium) activity.

In vivo MRI. Animal experiments were conducted in line with French Agriculture Ministry guidelines. The C57BL/6 healthy mice and tumor bearing mice received SPION hybridosomes $(1 \mathrm{mg}$ iron $/ \mathrm{kg}$ body weight, stabilized with PAA 450k) or SPION-AuNP hybridosomes $(0.5$ to $0.9 \mathrm{mg}$ iron/kg body weight, stabilized with PAA 450k or PAA 1.8k) by retro-orbital intravenous injection, after anesthesia with isoflurane. MR imaging sequences were recorded prior to the injection of the contrast agent, and 30 mins and $24 \mathrm{~h}$ after injection, using the $4.7 \mathrm{~T}$ (Brucker) small animal imager. Magnetic Resonance (MR) images were acquired using a Bruker Biospec 47/40 (Bruker Biospin, Wissembourg, France) with a 4.7 T magnet with a BG-06 gradient insert. Anesthetized animals were placed in a supine position in a $3.5-\mathrm{cm}$ bird-cage resonator. Images were acquired using a gradient echo (FLASH) sequence with $\mathrm{TR}=$ $300 \mathrm{~ms}, \mathrm{TE}=5 \mathrm{~ms}$, Flip Angle $=30^{\circ}, 4$ averages per phase-encoding step, a $212 \times 212$ matrix and FOV $=3.2 \times 3.2$. Fifteen $1 \mathrm{~mm}$ thick axial slices covering the mouse abdomen were acquired. Respiratory gating was used during the acquisition. A tube filled with water was used as reference. Respiratory triggering was performed using a highly sensitive pneumatic system placed under the mouse. Body temperature was maintained with a warming blanket.

2. Investigation of $\mathrm{THF} /$ water and $\mathrm{THF} /$ water/NP mixtures

a) $\mathrm{THF} /$ water mixtures

For dynamic light scattering measurements of THF/water mixture, great care was taken to avoid any impurities: water was filtered through a $0.2 \mu \mathrm{m}$ cellulose filter before use and THF was never in contact with any plastic material. Only glassware was used for all syringes, flasks and measurements cell. Dynamic light scattering (DLS) measurements of mixtures of THF and water were achieved for various volume fractions of water $\left(\varphi_{\mathrm{w}}\right)$ in the range $\varphi_{\mathrm{w}}=0.05-0.9$ (Figure $\mathrm{S} 1$ ). The relaxation times extracted from the correlograms are interpreted in terms of Brownian motion of spherical particles. Figure S1 shows that fluctuations of concentrations are measured over the whole range of compositions. As the fraction of water increases, faster dynamics is observed, corresponding to smaller length scale.

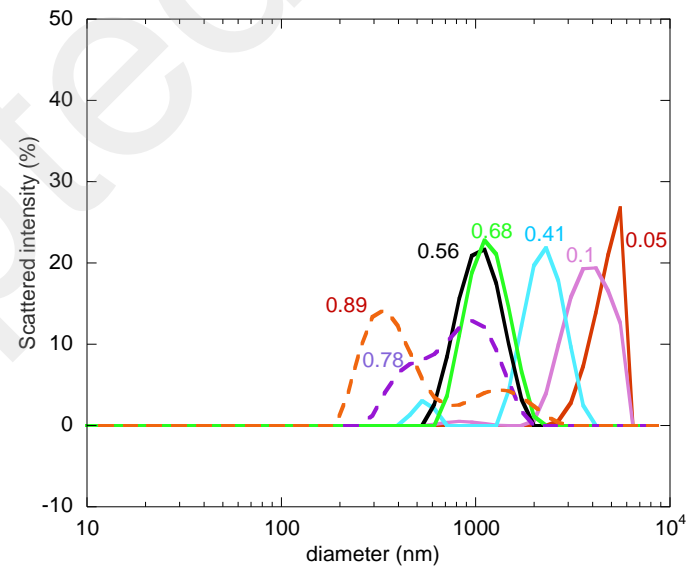

Figure S1: DLS measurements of THF/water mixtures. Volume fraction of water $\varphi_{w}$ as indicated on graph.

A mode corresponding to the Brownian motion of spherical objects of ca $250 \mathrm{~nm}$ can be observed at $\varphi_{\mathrm{w}}=0.78$ and $\varphi_{\mathrm{w}}=0.89$. Since DLS is sensitive to any fluctuation, the dynamic modes that were measured do not necessarily correspond to defined long-lived structures. Therefore, Nanoparticle Tracking Analysis (NTA) analysis was undertaken for compositions where the fastest correlation modes were observed $\left(\varphi_{\mathrm{w}}=0.8-0.9\right)$. NTA is a microscopic analysis of individual trajectories of spotlights scattered by scattering objects in solution. It was shown to accurately analyze the size distribution of polydisperse samples, which is not the case for DLS. Individual objects were clearly observed (Figure S2) using NTA. The size distribution profile shows a narrow distribution with a main mode at $\mathrm{D}_{\mathrm{h}}=110 \pm 35 \mathrm{~nm}$. 

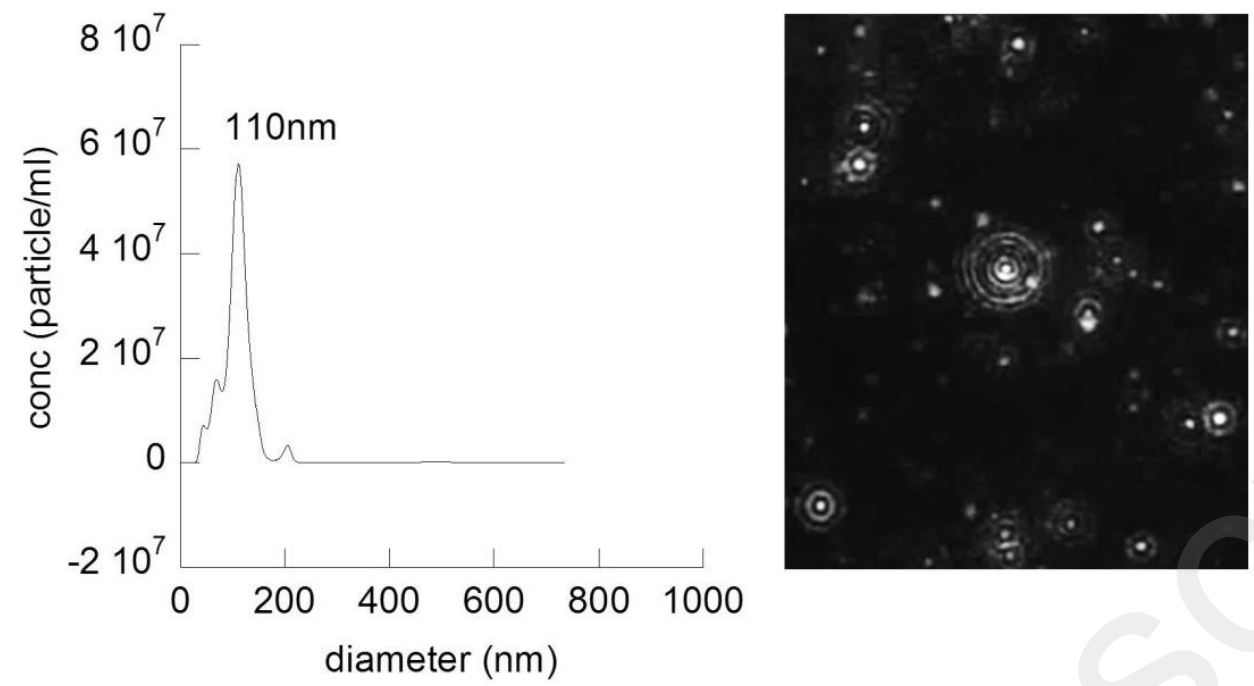

Figure S2: NTA analysis of a THF/water mixture for $\varphi_{w}=0.8$. Left: size distribution profile. Right: optical microscopy image of the scattered spotlights.

b) Investigation of water/THF/NP mixtures 


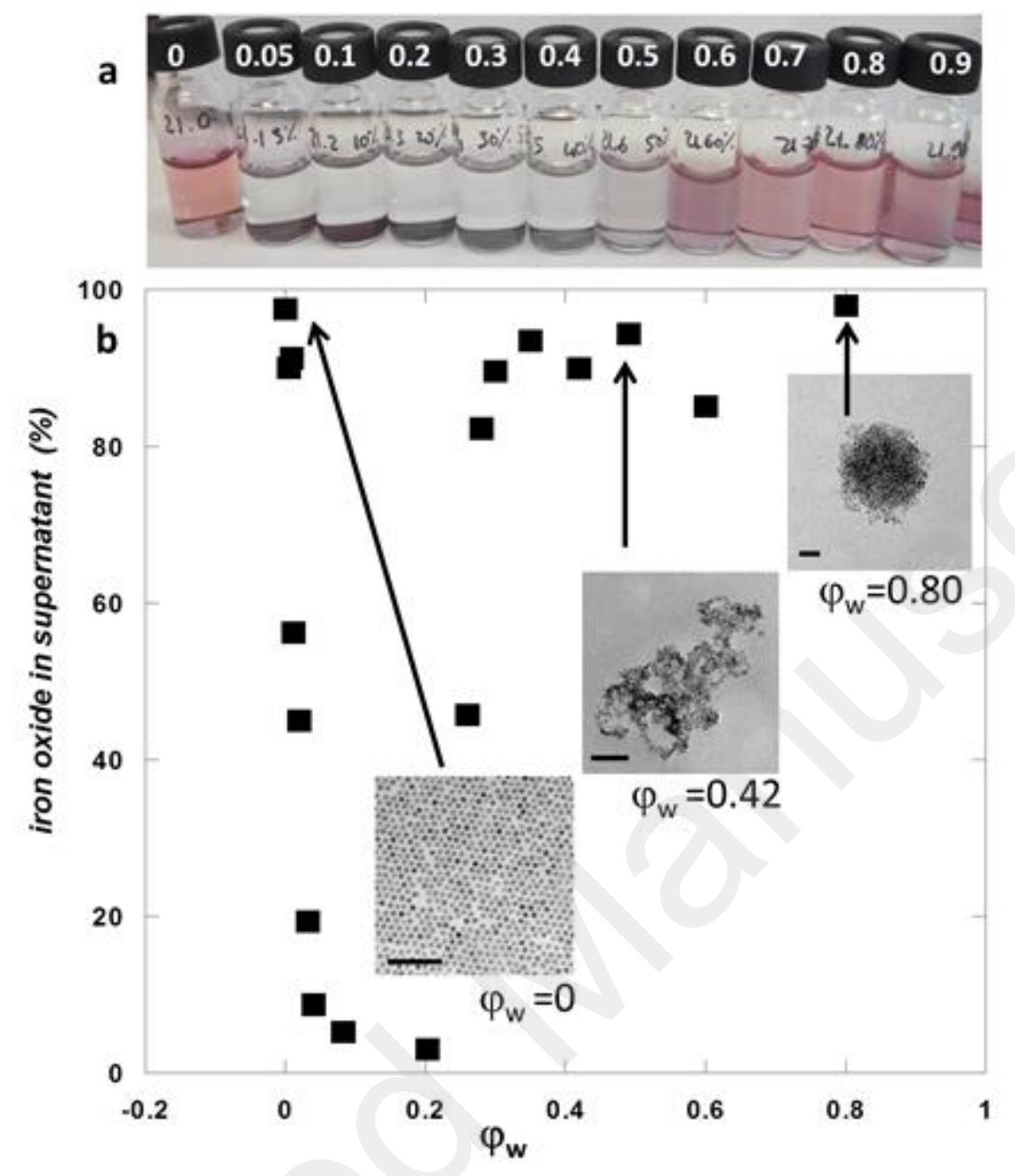

Figure S3: (a) Picture of solutions of AuNP in water/THF (the white figures on caps indicate the water volume fraction $\left.\varphi_{w}\right)$. (b) Stability of SPION in water/THF and TEM pictures obtained from the supernatants. Scale bars: 50 $\mathbf{m}$.

c) Effects of nanoparticles over THF/water mixtures (DLS) 

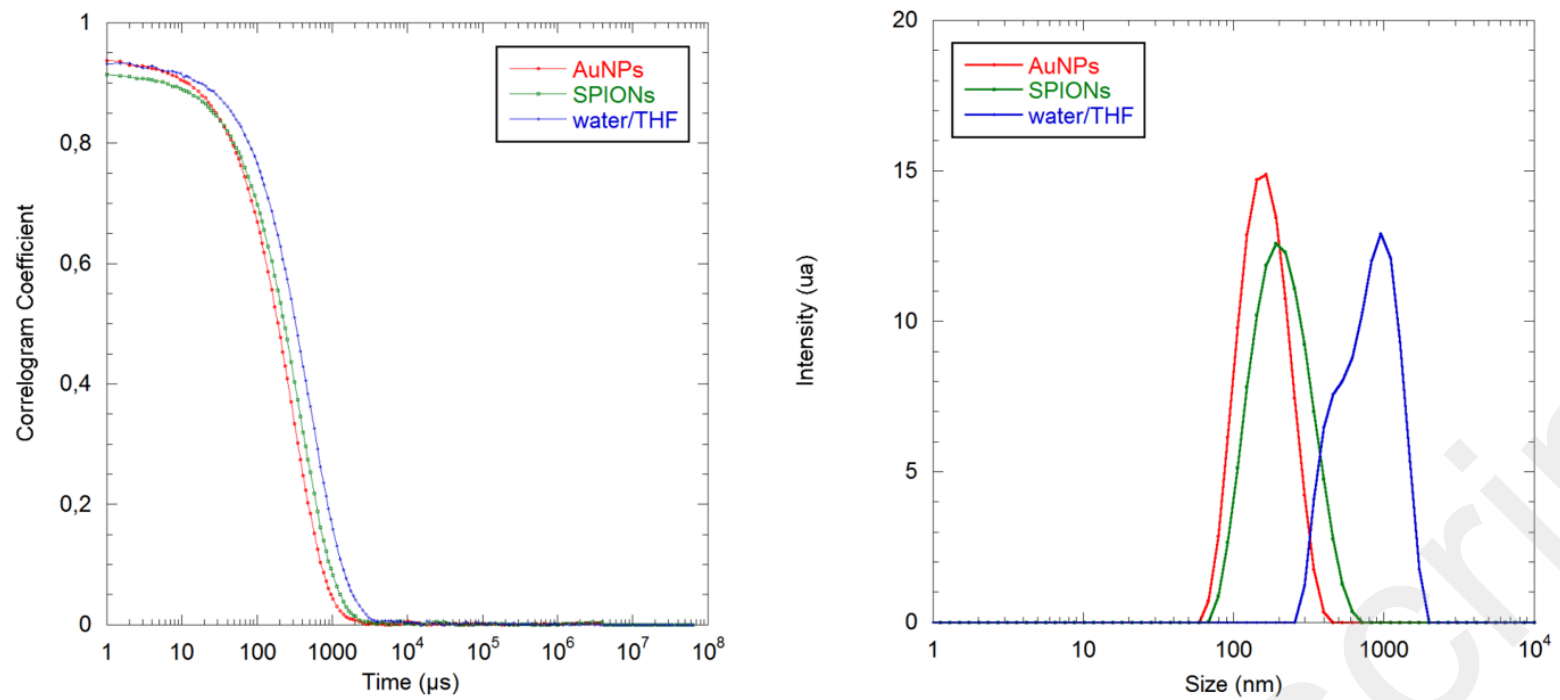

Figure S4: Correlograms (a) and size distribution (b) (by scattered intensity) from DLS measurements of water/THF mixtures $\left(\varphi_{w}=0.8\right)$ in absence of NP (blue) and in presence of SPION (green) and AuNP (red).

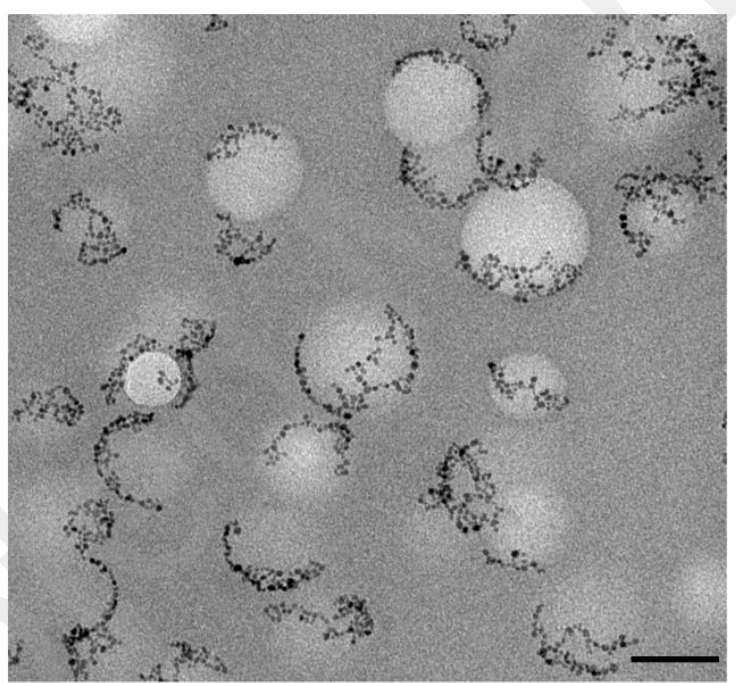

Figure S5: TEM picture of assemblies of SPION at low nanoparticle concentration, in order to evidence the templating effect of liquid droplets. [Fe] $=5 \mu \mathrm{g} / \mathrm{ml}$; polymer: PAA450k. Scale bar: $100 \mathrm{~nm}$

3. Energy dispersive X-ray spectroscopy (EDX)

EDX analysis was coupled to TEM observation to demonstrate the colocalization of iron and gold in a single AuNP/SPION hybridosome. 


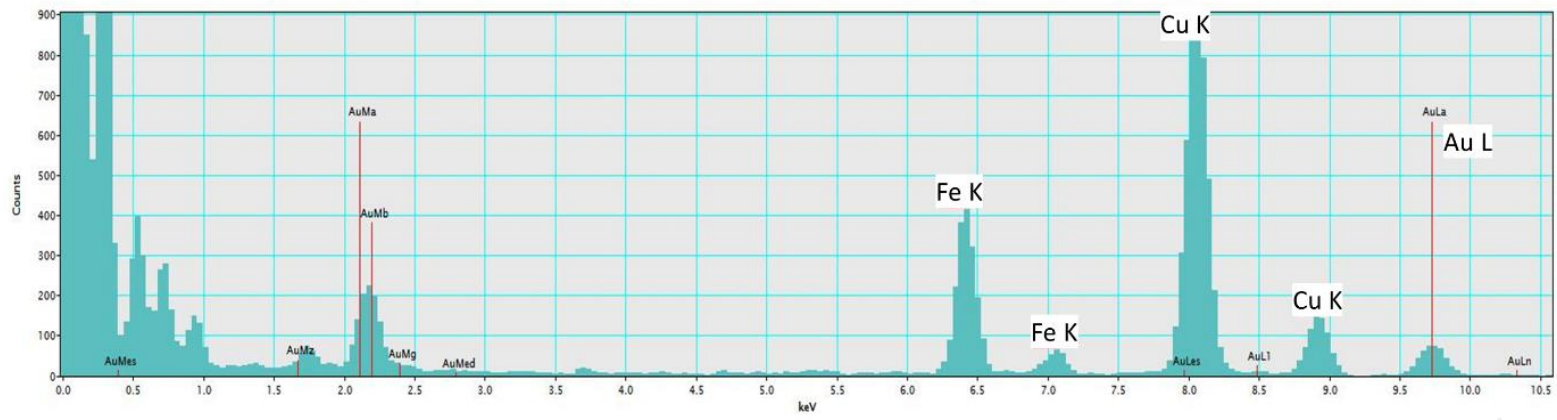

Figure S6: Energy dispersive X-ray spectroscopy of a single AuNP/SPION hybridosome showing the co-localization of iron and gold.

4. Fluo-magnetic hybridosomes

The magnetic SPION hybridosomes could also be loaded with a fluorescent BODIPY dye, simply by dissolving the dye into the THF solution at the initial stage of the fabrication process. The selected dye (BODIPY 1, Figure S7) is not soluble in water and fluoresces both in its dissolved and aggregated states. Two cycles of magnetic separation and washing with water were achieved to obtain bright fluorescent hybridosomes and a dye free supernatant as demonstrated by absorbance and emission measurements. Figure S7a shows the absorbance and emission spectra of the supernatant after the two cycles of washing, and Figure S7b shows the fluorescent hybridosomes after redispersion in water. No significant leakage of the dye was observed over months.

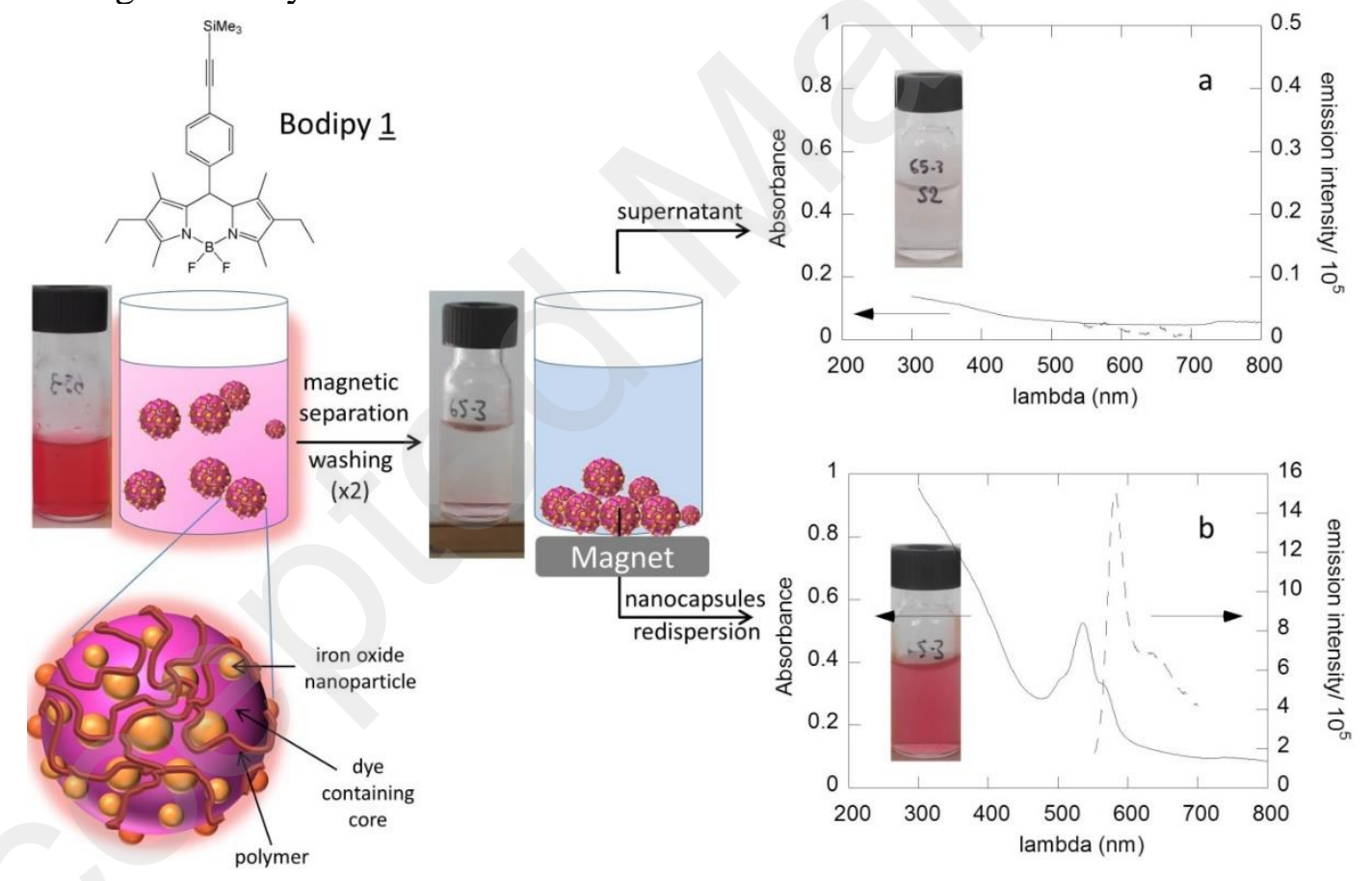

Figure S7: Elaboration of magnetic and fluorescent hybridosomes: chemical structure of the BODIPY $\underline{1}$. (a) Absorbance and emission spectra of the supernatant after removal of the fluo-magnetic hybridosomes using magnetic separation. (b) Absorbance and emission spectra of the suspension after redispersion of the hybridosomes in water.

\section{Transversal relaxivities}

The transversal relaxivities of SPION and SPION/AuNP hybridosomes stabilized with PAA or PEGPAA were measured in water using a $4.7 \mathrm{~T}$ magnetic resonance (MR) scanner (Figure S8). The measured values were $\mathrm{r}_{2}=274 \pm 35 \mathrm{mM}^{-1} \cdot \mathrm{s}^{-1}, 285 \pm 37 \mathrm{mM}^{-1} \cdot \mathrm{s}^{-1}$ and $257 \pm 15 \mathrm{mM}^{-1} \cdot \mathrm{s}^{-1}$ for the SPION/PAA, SPION/PEG-PAA, and SPION/AuNP PAA hybridosomes, respectively. All measured values compare Feraspin ${ }^{\mathrm{TM}}$, a commercial contrast agent for pre-clinical imaging, measured in the same conditions $\left(\mathrm{r}_{2}=307 \pm 80 \mathrm{mM}^{-1} \cdot \mathrm{s}^{-1}\right)$. The choice of the polymer, PAA or PEG-PAA, has no significant 
effect on the relaxivity of SPION hybridosomes in water. Mixed SPION/AuNP hybridosomes show a sligthly lower relaxivity, most probably due to a lesser extent of magnetic coupling between SPION.
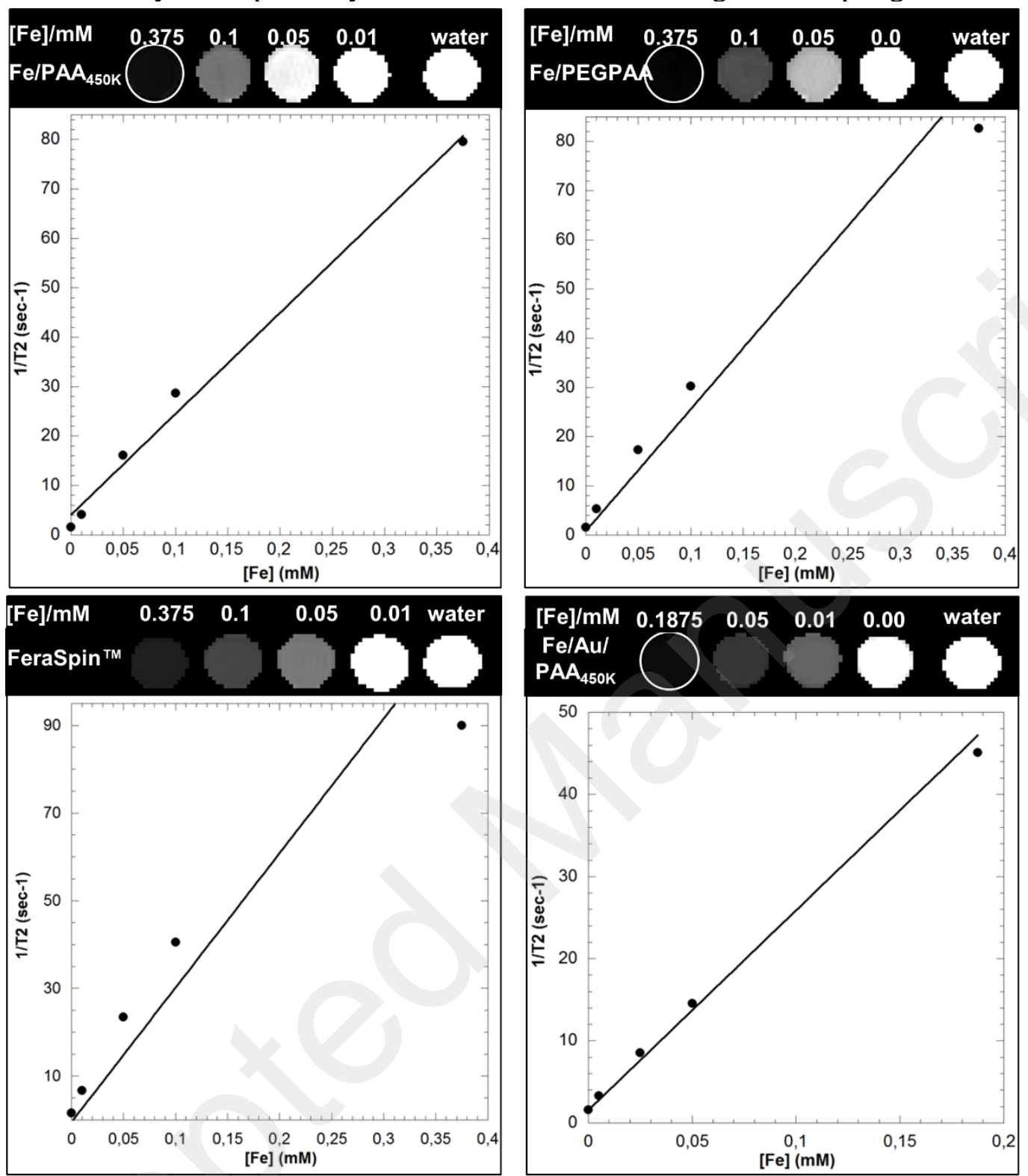

Figure S8: MR images obtained at $4.7 \mathrm{~T}$ of phantoms containing hybridosomes solutions of decreasing concentrations of (iron concentration as indicated) and graphs of reciprocal measured relaxation times vs iron concentrations for hybridosomes (a) SPION/PAA450k, (b)SPION/PEG-PAA, (c)SPION/AuNP/PAA450k and (d) commercial contrast agent Feraspin ${ }^{\mathrm{TM}}$ XXL.

\section{In vivo MRI}



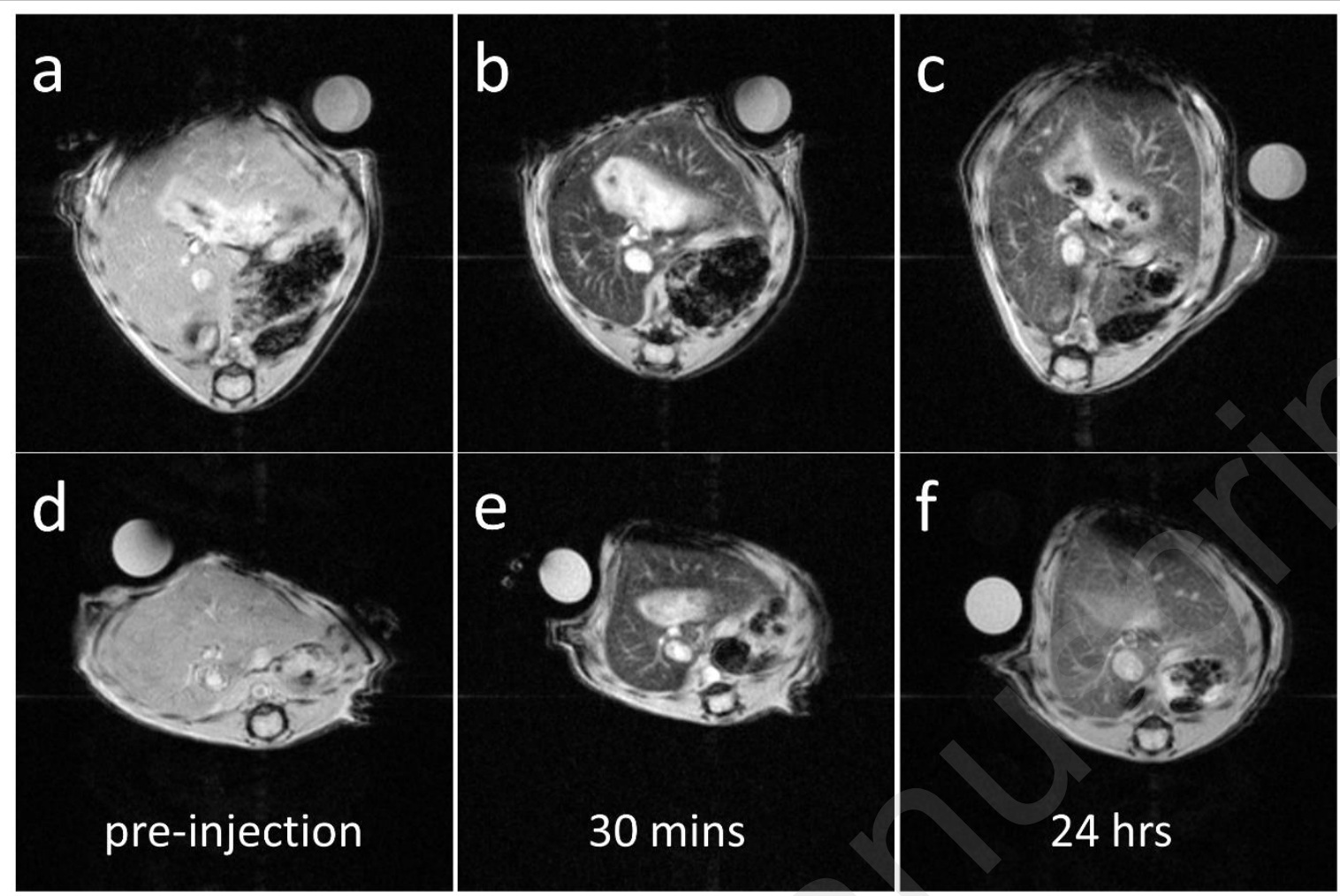

Figure S9: $\mathrm{T}_{2} *$-weighted MR images of healthy bearing mice injected with SPION hybridosomes stabilized with PAA450k (a-c) or PEG-PAA (d-f) and acquired at $t=0 ; 30$ min and $24 \mathrm{~h}$ after intravenous injection, showing a clear extinction of the liver.

\section{In vitro toxicity}




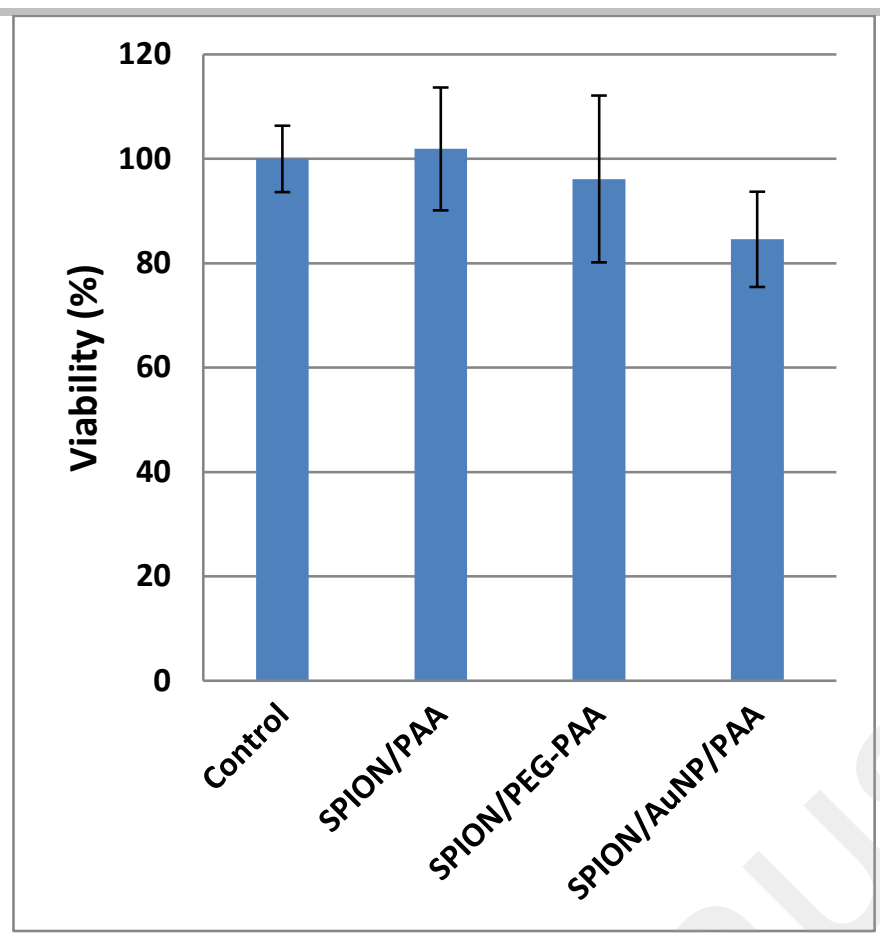

Figure S10: Viability of HEK cells after incubation $48 \mathrm{~h}$ with the magnetic SPION ([Fe]=100 $\left.\mu \mathrm{g} / 10^{6} \mathrm{cells}\right)$ or SPION/AuNP $\left([\mathrm{Fe}]=[\mathrm{Au}]=100 \mu \mathrm{g} / 10^{6} \mathrm{cells}\right)$ hybridosomes used as MRI contrast agents.

8. TEM tomography of hybridosomes

Movie S1 shows animated slab of images collected at tilted intervals.

[1] G. Casterou, V. Collière, P. Lecante, Y. Coppel, P.-A. Eliat, F. Gauffre, M. L. Kahn, Chemistry 2015, 21, 18855-61.

[2] J. Nayak, M. Aralaguppi, B. V. Naidu, T. Aminabhavi, J. Chem. Eng. Data 2004, 49, 468-474. 